\title{
SHARP LIOUVILLE THEOREMS
}

\author{
SALVADOR VILLEGAS
}

\begin{abstract}
Consider the equation $\operatorname{div}\left(\varphi^{2} \nabla \sigma\right)=0$ in $\mathbb{R}^{N}$, where $\varphi>$ 0 . Berestycki, Caffarelli and Nirenberg 4] proved that if there exists $C>0$ such that $\int_{B_{R}}(\varphi \sigma)^{2} \leq C R^{2}$ for every $R \geq 1$ then $\sigma$ is necessarily constant. In this paper we provide necessary and sufficient conditions on $0<\Psi \in C([1, \infty))$ for which this result remains true if we replace $R^{2}$ with $\Psi(R)$ in any dimension $N$. In the case of the convexity of $\Psi$ for large $R>1$ and $\Psi^{\prime}>0$, this condition is equivalent to $\int_{1}^{\infty} \frac{1}{\Psi^{\prime}}=\infty$.
\end{abstract}

\section{INTRODUCTION AND MAIN RESULTS}

In 1978 E. De Giorgi [5] made the following conjecture:

Conjecture. Let $u \in C^{2}\left(\mathbb{R}^{N}\right)$ be a bounded solution of the Allen-Cahn equation $-\Delta u=u-u^{3}$ which is monotone in one direction (for instance $\partial u / \partial_{x_{N}}>0$ in $\mathbb{R}^{N}$ ). Then $u$ is a 1-dimensional function (or equivalently, all level sets $\{u=s\}$ of $\mathrm{u}$ are hyperplanes), at least if $N \leq 8$.

This conjecture was proved in 1997 for $N=2$ by Ghoussoub and Gui [8], and in 2000 for $N=3$ by Ambrosio and Cabré [2] . In dimensions $N \geq 9$, del Pino, Kowalczyk, and Wei [6] established that the conjecture does not hold, as suggested in De Giorgi's original statement. The conjecture remains still open for dimensions $4 \leq N \leq 8$.

In the proof of the conjecture for $N \leq 3$, it is used the following Liouvilletype theorem due to H. Berestycki, L. Caffarelli and L. Nirenberg [4]:

Theorem 1.1. Let $\varphi \in L_{l o c}^{\infty}\left(\mathbb{R}^{N}\right)$ be a positive function. Assume that $\sigma \in$ $H_{\text {loc }}^{1}\left(\mathbb{R}^{N}\right)$ satisfies $\sigma \operatorname{div}\left(\varphi^{2} \nabla \sigma\right) \geq 0$ in $\mathbb{R}^{N}$ in the distributional sense. For every $R>0$, let $B_{R}=\{|x|<R\}$ and assume that there exists a constant independent of $R$ such that

$$
\int_{B_{R}}(\varphi \sigma)^{2} d x \leq C R^{2} \quad \text { for every } R \geq 1 .
$$

Then $\sigma$ is constant.

To deduce the conjecture for $N \leq 3$ from this theorem, the authors made the following reasoning: if $u$ is a solution in De Giorgi's conjecture, consider the functions $\varphi:=\partial u / \partial_{x_{N}}>0$ and $\sigma_{i}:=\partial_{x_{i}} u / \partial_{x_{N}} u$, for $i=1, \ldots, N-1$.

The author has been supported by the Ministerio de Ciencia, Innovación y Universidades of Spain PGC2018-096422-B-I00 and by the Junta de Andalucía FQM-116. 
Since both $\partial_{x_{i}} u$ and $\varphi$ solves the same linear equation $-\Delta v=\left(1-3 u^{2}\right) v$, an easy computation shows that $\operatorname{div}\left(\varphi^{2} \nabla \sigma_{i}\right)=0$. In dimensions $N \leq 3$ it is proved that there exists $C>0$ such that $\int_{B_{R}}|\nabla u|^{2} d x \leq C R^{2}$, for every $R \geq 1$. Applying Theorem 1.1 gives $\sigma_{i}$ is constant for every $i=1, \ldots, N-1$. It follows easily that $u$ is a one-dimensional function. Observe that in the previous reasoning it is only used $\operatorname{div}\left(\varphi^{2} \nabla \sigma_{i}\right)=0$, which is a an stronger condition than $\sigma_{i} \operatorname{div}\left(\varphi^{2} \nabla \sigma_{i}\right) \geq 0$.

Motivated by the useful application of Liouville-type theorems to these kind of problems, a natural question is to find functions $0<\Psi \in C([1, \infty))$, for which Theorem 1.1 remains true if we replace $C R^{2}$ with $\Psi(R)$. In this way, we make the following definitions:

Property (P). We say that a function $0<\Psi \in C([1, \infty))$ satisfies (P) if it has the following property: if $\varphi \in L_{l o c}^{\infty}\left(\mathbb{R}^{N}\right)$ is a positive function, $\sigma \in H_{l o c}^{1}\left(\mathbb{R}^{N}\right)$ satisfies

$$
\sigma \operatorname{div}\left(\varphi^{2} \nabla \sigma\right) \geq 0 \quad \text { in } \mathbb{R}^{N}
$$

in the distributional sense and

$$
\int_{B_{R}}(\varphi \sigma)^{2} d x \leq \Psi(R) \text { for every } R \geq 1
$$

then $\sigma$ is necessarily constant.

Property ( $\left.\mathbf{P}^{\prime}\right)$. We say that a function $0<\Psi \in C([1, \infty))$ satisfies $\left(\mathrm{P}^{\prime}\right)$ if it has the following property: if $\varphi \in L_{l o c}^{\infty}\left(\mathbb{R}^{N}\right)$ is a positive function, $\sigma \in H_{l o c}^{1}\left(\mathbb{R}^{N}\right)$ satisfies

$$
\operatorname{div}\left(\varphi^{2} \nabla \sigma\right)=0 \quad \text { in } \mathbb{R}^{N}
$$

in the distributional sense and

$$
\int_{B_{R}}(\varphi \sigma)^{2} d x \leq \Psi(R) \text { for every } R \geq 1
$$

then $\sigma$ is necessarily constant.

Note that, a priori, the definitions of properties $(\mathrm{P})$ and $\left(\mathrm{P}^{\prime}\right)$ depend on the dimension $N$. We will show that, in fact, this is not so: if a function $0<\Psi \in C([1, \infty))$ satisfies $(\mathrm{P})$ (resp. $\left.\left(\mathrm{P}^{\prime}\right)\right)$ in some dimension $N_{0}$, then it satisfies $(\mathrm{P})$ (resp. $\left.\left(\mathrm{P}^{\prime}\right)\right)$ in any dimension $N$.

It is obvious that property $(\mathrm{P})$ is stronger than property $\left(\mathrm{P}^{\prime}\right)$. In fact, in this paper we will prove that they are equivalent.

With this notation, Theorem 1.1 says that the function $C R^{2}$ satisfies (P) for every $C>0$. In [1] the authors formulated the following problem: What is the optimal (maximal) exponet $\gamma_{N}$ such that $C R^{\gamma_{N}}(C>0)$ satisfies $\left(\mathrm{P}^{\prime}\right)$ ? 
In [3] it is proved that $\gamma_{N}<N$ when $N \geq 3$. Also, a sharp choise in the counterexamples of [8] shows that $\gamma_{N}<2+2 \sqrt{N-1}$ for $N \geq 7$. Recently, Moradifam [9] proved that $\gamma_{N}<3$ when $N \geq 4$. Finally, in a recent work 12 the author has proven that $\gamma_{N}=2$ for every $N \geq 1$. In other words, the functions $C R^{k}$ do not satisfy ( $\left.\mathrm{P}^{\prime}\right)$ for every $k>2$ and $C>0$. On the other hand, the sharpness of the exponent 2 for condition $(\mathrm{P})$ was proved by Gazzola 7 .

Moschini [10] proved that $C R^{2}(1+\log R)$ satisfies (P) for every $C>0$. By a classical example [11] it is obtained that $R^{2}(1+\log R)^{2}$ does not satisfy (P) in dimension $N=2$.

All the results previously exposed are covered by the following theorems:

Theorem 1.2. Suppose $0<\Psi \in C([1, \infty))$. The following conditions are equivalent:

i) $\Psi$ satisfies $(P)$.

ii) $\Psi$ satisfies $\left(P^{\prime}\right)$.

iii) $\int_{1}^{\infty} \frac{1}{h^{\prime}}=\infty$, for every nondecreasing function $0 \leq h \in C([1, \infty))$ satisfying $h \leq \Psi$ in $[1, \infty)$.

Note that if $0<\Psi \in C^{1}([1, \infty))$ satisfy $\Psi^{\prime}>0$ in $[1,+\infty)$, the we can take $h=\Psi$ in iii), obtaining that $\int_{1}^{\infty} 1 / \Psi^{\prime}=\infty$ is a necessary condition to have i) and ii), but not sufficient (see Remark 2 below). The next theorem shows that, under convexity conditions on $\Psi$, this is also a sufficient condition to obtain i) and ii).

Theorem 1.3. Suppose $0<\Psi \in C^{1}([1, \infty))$ satisfy $\Psi^{\prime}>0$ in $[1,+\infty)$ and $\Psi$ is convex in $\left[R_{0},+\infty\right)$ for some $R_{0}>1$. The following conditions are equivalent:

i) $\Psi$ satisfies $(P)$.

ii) $\Psi$ satisfies $\left(P^{\prime}\right)$.

iii') $\int_{1}^{\infty} \frac{1}{\Psi^{\prime}}=\infty$.

Remark 1. For general $0<\Psi \in C([1, \infty))$, it is possible to prove that if $\liminf _{x \rightarrow \infty} \Psi(x) / x^{2}<+\infty$ then $\Psi$ satisfy $(\mathrm{P})$ and $\left(\mathrm{P}^{\prime}\right)$. Therefore, we can restrict our attention to the case $\lim _{x \rightarrow \infty} \Psi(x) / x^{2}=+\infty$. Thus, the condition of convexity of $\Psi$ in Theorem 1.3 seems natural and not too restrictive.

To see that $\liminf _{x \rightarrow \infty} \Psi(x) / x^{2}<+\infty$ implies $\Psi$ satisfy $(\mathrm{P})$ and (P') we will apply Theorem 1.2. Suppose that there exists a divergent sequence $\left\{R_{n}\right\}$ and a real number $C>0$ such that $\Psi\left(R_{n}\right) \leq C R_{n}^{2}, n \geq 1$ and take a nondecreasing function $0 \leq h \in C([1, \infty))$ satisfying $h \leq \Psi$ in $[1, \infty)$. Our purpose is to obtain $\int_{1}^{\infty} 1 / h^{\prime}=\infty$. To this end, take an arbitrary $R>1$ and consider $n_{0} \in \mathbb{N}$ such that $R_{n}>R$ for every $n \geq n_{0}$. Then

$$
R_{n}-R=\int_{R}^{R_{n}} \sqrt{h^{\prime}} \frac{1}{\sqrt{h^{\prime}}} \leq\left(\int_{R}^{R_{n}} h^{\prime}\right)^{1 / 2}\left(\int_{R}^{R_{n}} \frac{1}{h^{\prime}}\right)^{1 / 2}
$$




$$
\begin{gathered}
=\left(h\left(R_{n}\right)-h(R)\right)^{1 / 2}\left(\int_{R}^{R_{n}} \frac{1}{h^{\prime}}\right)^{1 / 2} \leq\left(\Psi\left(R_{n}\right)\right)^{1 / 2}\left(\int_{R}^{R_{n}} \frac{1}{h^{\prime}}\right)^{1 / 2} \\
\leq C^{1 / 2} R_{n}\left(\int_{R}^{\infty} \frac{1}{h^{\prime}}\right)^{1 / 2},
\end{gathered}
$$

for every $n \geq n_{0}$. Hence

$$
\int_{R}^{\infty} \frac{1}{h^{\prime}} \geq \frac{\left(R_{n}-R\right)^{2}}{C R_{n}^{2}}
$$

for $n \geq n_{0}$. Taking limit as $n$ tends to infinity we deduce

$$
\int_{R}^{\infty} \frac{1}{h^{\prime}} \geq \frac{1}{C}
$$

Since $R>1$ is arbitrary we conclude $\int_{1}^{\infty} 1 / h^{\prime}=\infty$, which is the desired conclusion.

Remark 2. As said before, if $0<\Psi \in C^{1}([1, \infty))$ satisfy $\Psi^{\prime}>0$ in $[1,+\infty)$, it is obvious from Theorem 1.2 that the condition $\int_{1}^{\infty} 1 / \Psi^{\prime}=\infty$ is necessary to have i) and ii). To show that this is not sufficient, it suffices to construct functions $\Psi, h \in C^{\infty}([1, \infty))$ satisfying $0<h<\Psi$ and $0<\Psi^{\prime}, h^{\prime}$ in $[1, \infty)$ such that $\int_{1}^{\infty} 1 / \Psi^{\prime}=\infty$ and $\int_{1}^{\infty} 1 / h^{\prime}<\infty$.

To do this, for every integer $n \geq 1$ define the function $f_{n}:[n, n+1 / 2] \rightarrow \mathbb{R}$ by

$$
f_{n}(x):=7 n x+n^{3}, n \leq x \leq n+1 / 2 .
$$

Clearly

$f_{n}(n+1 / 2)=7 n(n+1 / 2)+n^{3}<f_{n+1}(n+1)=7(n+1)^{2}+(n+1)^{3}$, for every $n \geq 1$.

Hence, there exists $0<\Psi \in C^{\infty}\left([1,+\infty)\right.$ satisfying $\Psi^{\prime}>0$ and $\Psi(x)=$ $f_{n}(x)$ for every $n \leq x \leq n+1 / 2$ and $n \geq 1$.

It follows that

$$
\int_{1}^{\infty} \frac{1}{\Psi^{\prime}} \geq \sum_{n \geq 1} \int_{n}^{n+1 / 2} \frac{1}{\Psi^{\prime}}=\sum_{n \geq 1} \frac{1}{14 n}=\infty .
$$

Then $\int_{1}^{\infty} 1 / \Psi^{\prime}=\infty$. On the other hand, take an arbitrary $x \geq 1$. Then there exists an integer $n \geq 1$ such that $n \leq x<n+1$. Thus

$$
\Psi(x) \geq \Psi(n)=7 n^{2}+n^{3} \geq(n+1)^{3}>x^{3} .
$$

Therefore, taking $h(x)=x^{3}$ we have $\int_{1}^{\infty} 1 / h^{\prime}<\infty$, which is our claim. 


\section{Proof of Theorem 1.2.}

\section{Proof of Theorem 1.2.}

It is evident that i) $\Rightarrow$ ii). Therefore we shall have established the theorem if we prove iii $\Rightarrow$ i) and ii) $\Rightarrow$ iii).

\section{Proof of $i i i) \Rightarrow i$ )}

Suppose that $0<\Psi \in C([1, \infty))$ satisfies iii) and $\int_{B_{R}}(\varphi \sigma)^{2} \leq \Psi(R)$ for every $R \geq 1$ where $\varphi \in L_{l o c}^{\infty}\left(\mathbb{R}^{N}\right)$ is a positive function and $\sigma \in H_{l o c}^{1}\left(\mathbb{R}^{N}\right)$ satisfies (1.1) in the distributional sense. Our purpose is to obtain that $\sigma$ is constant.

If inf $\Psi=0$ then there exists a divergent sequence $\left\{R_{n}\right\}$ such that $\Psi\left(R_{n}\right)$ tends to 0 as $n$ tends to $\infty$. Thus $\int_{B_{R_{n}}}(\varphi \sigma)^{2}$ also tends to 0 , which implies $\sigma=0$.

Otherwise, let $0<m:=\inf \Psi$ and consider the function

$$
h(r):=\frac{1}{2} \int_{B_{r}}(\varphi \sigma)^{2}+\frac{1}{2} m\left(1-e^{-r}\right) \quad r \geq 1 .
$$

Clearly, $h \leq 1 / 2 \Psi+1 / 2 m \leq \Psi$ in $[1, \infty)$ and $h$ is a positive continuous and nondecreasing function satisfying

$$
h^{\prime}(r)=\frac{1}{2}\left(\int_{|x|=r}(\varphi \sigma)^{2}\right)+\frac{1}{2} m e^{-r},
$$

for almost every $r>1$. From this $\int_{1}^{\infty} 1 / h^{\prime}=\infty$. Taking into account that $1 / h^{\prime}(r) \leq 2 e^{r} / m$ for almost every $r>1$ we have $1 / h^{\prime} \in L_{l o c}^{\infty}([1, \infty))$. Thus

$$
\int_{R}^{\infty} \frac{1}{h^{\prime}}=\infty, \text { for every } R>1
$$

Now, for arbitrary $1<R_{1}<R_{2}$ define in the ball $B_{R_{2}}$ the radial function $\eta$ by

$$
\eta(r):= \begin{cases}1 & \text { if } 0 \leq r \leq R_{1} \\ \frac{\int_{r}^{R_{2}} \frac{1}{h^{\prime}}}{\int_{R_{1}}^{R_{2}} \frac{1}{h^{\prime}}} & \text { if } R_{1}<r \leq R_{2}\end{cases}
$$

for every $r=|x| \leq R_{2}$. Multiplying (1.1) by $\eta^{2}$ and integrating by parts in $B_{R_{2}}$, we obtain

$$
\begin{gathered}
\int_{B_{R_{2}}} \eta^{2} \varphi^{2}|\nabla \sigma|^{2} \leq-2 \int_{B_{R_{2}}} \eta \varphi^{2} \sigma \nabla \eta \cdot \nabla \sigma \\
\leq 2\left(\int_{B_{R_{2}}} \eta^{2} \varphi^{2}|\nabla \sigma|^{2}\right)^{1 / 2}\left(\int_{B_{R_{2}}} \varphi^{2} \sigma^{2}|\nabla \eta|^{2}\right)^{1 / 2} .
\end{gathered}
$$


Therefore

$$
\int_{B_{R_{2}}} \eta^{2} \varphi^{2}|\nabla \sigma|^{2} \leq 4 \int_{B_{R_{2}}} \varphi^{2} \sigma^{2}|\nabla \eta|^{2} .
$$

Thus

$$
\begin{gathered}
\int_{B_{R_{1}}} \varphi^{2}|\nabla \sigma|^{2} \leq \int_{B_{R_{2}}} \eta^{2} \varphi^{2}|\nabla \sigma|^{2} \leq 4 \int_{B_{R_{2}}} \varphi^{2} \sigma^{2}|\nabla \eta|^{2} \\
=4 \int_{R_{1}}^{R_{2}} \eta^{\prime}(r)^{2}\left(\int_{|x|=r}(\varphi \sigma)^{2}\right) d r \leq 4 \int_{R_{1}}^{R_{2}} \eta^{\prime}(r)^{2} 2 h^{\prime}(r) d r \\
=\frac{8}{\left(\int_{R_{1}}^{R_{2}} \frac{1}{h^{\prime}}\right)^{2}} \int_{R_{1}}^{R_{2}} \frac{1}{h^{\prime}(r)^{2}} h^{\prime}(r) d r=\frac{8}{\int_{R_{1}}^{R_{2}} \frac{1}{h^{\prime}}} .
\end{gathered}
$$

Fix $R_{1}>1$. Applying (2.1) and taking limit in the above inequality as $R_{2}$ tends to $\infty$ we obtain

$$
\int_{B_{R_{1}}} \varphi^{2}|\nabla \sigma|^{2}=0
$$

Since $R_{1}>1$ is arbitrary, $\sigma$ is constant, which is the desired conclusion.

\section{Proof of $i i) \Rightarrow$ iii)}

Suppose that iii) does not hold. That is, there exists a nondecreasing function $0 \leq h \in C([1, \infty))$ satisfying $h \leq \Psi$ in $[1, \infty)$ and $\int_{1}^{\infty} \frac{1}{h^{\prime}}<\infty$. The proof is completed by constructing a positive function $\varphi \in L_{l o c}^{\infty}\left(\mathbb{R}^{N}\right)$ and a noncontant function $\sigma \in H_{l o c}^{1}\left(\mathbb{R}^{N}\right)$ satisfying (1.2) in the distributional sense and $\int_{B_{R}}(\varphi \sigma)^{2} \leq \Psi(R)$ for every $R \geq 1$.

First of all, note that $0<\lim _{r \rightarrow \infty} h(r) \leq \liminf _{r \rightarrow \infty} \Psi(r)$. Since $\Psi>0$ in $[1, \infty)$, we have that $0<m:=\inf \Psi$. Consider the odd function $\mu: \mathbb{R} \rightarrow \mathbb{R}$ such that

$$
\mu(r):= \begin{cases}\frac{m}{2}\left(1-e^{-r}\right) & \text { if } 0 \leq r \leq 1 \\ \frac{1}{2} \int_{1}^{r} \min \left\{h^{\prime}(s), s^{2}\right\} d s+\frac{m}{2}\left(1-e^{-r}\right) & \text { if } 1<r\end{cases}
$$

Clearly $\mu$ is continuous and increasing in $\mathbb{R}$ and satisfies, almost everywhere, that 


$$
\mu^{\prime}(r):= \begin{cases}\frac{m}{2} e^{-|r|} & \text { if } 0 \leq|r| \leq 1 \\ \frac{1}{2} \min \left\{h^{\prime}(|r|), r^{2}\right\}+\frac{m}{2} e^{-|r|} & \text { if } 1<|r|\end{cases}
$$

Therefore

$$
0<\frac{1}{\mu^{\prime}(r)}<\frac{2}{\min \left\{h^{\prime}(r), r^{2}\right\}} \leq \frac{2}{h^{\prime}(r)}+\frac{2}{r^{2}}, \text { for every } r>1 .
$$

Hence $1 / \mu^{\prime} \in L^{1}(1, \infty)$ and it follows immediately $1 / \mu^{\prime} \in L^{1}(\mathbb{R})$. For this reason, taking any $0<H \in C^{\infty}\left(\mathbb{R}^{N-1}\right)$ satisfying $\int_{\mathbb{R}^{N-1}} H^{2}=1 / 2$, we can define the functions $\varphi, \sigma: \mathbb{R}^{N} \rightarrow \mathbb{R}$ by

$$
\begin{gathered}
\varphi\left(x_{1}, \ldots, x_{N}\right):=H\left(x_{1}, \ldots, x_{N-1}\right) \sqrt{\mu^{\prime}\left(x_{N}\right)} \int_{x_{N}}^{+\infty} \frac{d r}{\mu^{\prime}(r)}, \\
\sigma\left(x_{1}, \ldots, x_{N}\right):=\frac{1}{\int_{x_{N}}^{+\infty} \frac{d r}{\mu^{\prime}(r)}} .
\end{gathered}
$$

(If $N=1$, then define $\varphi(x)=\sqrt{\mu^{\prime}(x)} \int_{x}^{+\infty} \frac{d r}{\mu^{\prime}(r)} / \sqrt{2}$ and we apply the same reasonig that in the case $N>1$ ).

It is easy to check that

$$
0<\mu^{\prime}(r) \leq \frac{1}{2} r^{2}+\frac{m}{2} e^{-|r|}, \quad \frac{1}{\mu^{\prime}(r)} \leq \frac{2}{m} e^{|r|}, r \in \mathbb{R} .
$$

From the above it follows that $0<\varphi \in L_{\text {loc }}^{\infty}\left(\mathbb{R}^{N}\right)$ and $|\nabla \sigma| \in L_{\text {loc }}^{\infty}\left(\mathbb{R}^{N}\right)$. Thus $\sigma \in H_{l o c}^{1}\left(\mathbb{R}^{N}\right)$. Moreover, an easy computation shows that

$$
\begin{aligned}
& \nabla \sigma\left(x_{1}, \ldots, x_{N}\right)=\left(0, \ldots, 0, \frac{1}{\mu^{\prime}\left(x_{N}\right)\left(\int_{x_{N}}^{+\infty} \frac{d r}{\mu^{\prime}(r)}\right)^{2}}\right), \\
& \left(\varphi^{2} \nabla \sigma\right)\left(x_{1}, \ldots, x_{N}\right)=\left(0, \ldots, 0, H^{2}\left(x_{1}, \ldots, x_{N-1}\right)\right),
\end{aligned}
$$

which implies $\operatorname{div}\left(\varphi^{2} \nabla \sigma\right)=0$ in $\mathbb{R}^{N}$.

Finally taking into account that $B_{R} \subset \mathbb{R}^{N-1} \times(-R, R)$, we obtain for every $R \geq 1$

$$
\begin{gathered}
\int_{B_{R}}(\varphi \sigma)^{2} d x=\int_{B_{R}} H^{2}\left(x_{1}, \ldots, x_{N-1}\right) \mu^{\prime}\left(x_{N}\right) d x \\
\leq \int_{\mathbb{R}^{N-1}} H^{2} d\left(x_{1}, \ldots, x_{N-1}\right) \int_{-R}^{R} \mu^{\prime}(r) d r=\frac{1}{2}(\mu(R)-\mu(-R))=\mu(R)
\end{gathered}
$$




$$
\leq \frac{1}{2} \int_{1}^{R} h^{\prime}(s) d s+\frac{m}{2}\left(1-e^{-R}\right) \leq \frac{h(R)}{2}+\frac{m}{2} \leq \frac{\Psi(R)}{2}+\frac{\Psi(R)}{2}=\Psi(R),
$$

which completes the proof.

\section{Proof of Theorem 1.3}

Proposition 3.1. Let $\phi \in C^{1}([a, b])$ a convex function satisfying $\phi^{\prime}>0$ in $[a, b]$. Then

$$
\int_{a}^{b} \frac{1}{\phi^{\prime}} \leq \int_{a}^{b} \frac{1}{g^{\prime}}
$$

for every nondecreasing function $g \in C([a, b])$ satisfying $g(a)=\phi(a)$ and $g \leq \phi$ in $[a, b]$.

Moreover, equality holds if and only if $g=\phi$.

Lemma 3.2. Let $g \in C([a, b])$ a nondecreasing function. Let $p(x)=A x+B$, $A>0, B \in \mathbb{R}$ such that $g(a)=p(a), g(b) \leq p(b)$. Then

$$
\int_{a}^{b} \frac{1}{p^{\prime}} \leq \int_{a}^{b} \frac{1}{g^{\prime}}
$$

Moreover, equality holds if and only if $g=p$.

\section{Proof.}

If $\int_{a}^{b} 1 / g^{\prime}=\infty$ the lemma s trivial. Otherwise, applying Cauchy-Shwartz inequality we obtain

$$
b-a=\int_{a}^{b} \sqrt{g^{\prime}} \frac{1}{\sqrt{g^{\prime}}} \leq\left(\int_{a}^{b} g^{\prime}\right)^{1 / 2}\left(\int_{a}^{b} \frac{1}{g^{\prime}}\right)^{1 / 2}=(g(b)-g(a))^{1 / 2}\left(\int_{a}^{b} \frac{1}{g^{\prime}}\right)^{1 / 2} .
$$

Hence

$$
\int_{a}^{b} \frac{1}{g^{\prime}} \geq \frac{(b-a)^{2}}{g(b)-g(a)} \geq \frac{(b-a)^{2}}{p(b)-p(a)}=\int_{a}^{b} \frac{1}{p^{\prime}} .
$$

On the other hand, if equality holds then all the previous inequalities become equalities. This implies that $g(b)=p(b)$ and that $\sqrt{g^{\prime}}$ is a real multiple of $1 / \sqrt{g^{\prime}}$. That is, $g^{\prime}$ is constant and, since $g(a)=p(a), g(b)=p(b)$, we obtain $g=p$.

Lemma 3.3. Let $g \in C([a, b])$ a nondecreasing function. For $1 \leq i \leq m$ consider $p_{i}(x)=A_{i} x+B_{i}, A_{i}>0, B_{i} \in \mathbb{R}$; such that $p_{i}(a) \leq g(a)$. Define

$$
\bar{g}(x):=\max \left\{g(x), p_{1}(x), p_{2}(x), \ldots, p_{m}(x)\right\}, a \leq x \leq b .
$$

Then 


$$
\int_{a}^{b} \frac{1}{\bar{g}^{\prime}} \leq \int_{a}^{b} \frac{1}{g^{\prime}}
$$

Moreover, if $\int_{a}^{b} 1 / g^{\prime}<\infty$, then equality holds if and only if $g=\bar{g}$.

\section{Proof.}

Note that $\bar{g}$ is a nondecreasing continuous function in $[a, b]$. Therefore, the statement of the lemma has sense. If $\int_{a}^{b} 1 / g^{\prime}=\infty$ the lemma s trivial.

Hence, we will suppose in the rest of the proof that $\int_{a}^{b} 1 / g^{\prime}<\infty$. The proof is by induction on $m$.

We first prove the lemma for $m=1$. To do this, consider the open set $G=\left\{x \in(a, b): p_{1}(x)>g(x)\right\}$. If $G=\emptyset$, then $\bar{g}=g$ and the lemma follows. Otherwise, $G$ is the countable (possible finite) disjoint union of open intervals. That is, $G=\cup_{n \in X}\left(a_{n}, b_{n}\right)$, where $X \subset \mathbb{N}$ and $p_{1}\left(a_{n}\right)=g\left(a_{n}\right)$, $p_{1}\left(b_{n}\right) \geq g\left(b_{n}\right)$ for every $n \in X$. Then

$$
\int_{a}^{b} \frac{1}{g^{\prime}}-\int_{a}^{b} \frac{1}{\bar{g}^{\prime}}=\int_{G}\left(\frac{1}{g^{\prime}}-\frac{1}{p_{1}^{\prime}}\right)=\sum_{n \in X} \int_{a_{n}}^{b_{n}}\left(\frac{1}{g^{\prime}}-\frac{1}{p_{1}^{\prime}}\right) .
$$

Applying Lemma 3.2 in each interval $\left(a_{n}, b_{n}\right)$ we conclude the lemma for the case $m=1$.

We now proceed by induction. Suppose that the lemma holds for $m-1 \geq 1$ and we will prove that it holds for $m$. Define

$$
h(x):=\max \left\{g(x), p_{1}(x), p_{2}(x), \ldots, p_{m-1}(x)\right\}, a \leq x \leq b .
$$

By hypothesis of induction we have

$$
\int_{a}^{b} \frac{1}{h^{\prime}} \leq \int_{a}^{b} \frac{1}{g^{\prime}}
$$

On the other hand, note that

$\bar{g}(x):=\max \left\{g(x), p_{1}(x), p_{2}(x), \ldots, p_{m}(x)\right\}=\max \left\{h(x), p_{m}(x)\right\}, a \leq x \leq b$.

It is easily seen that $h$ is a continuous nondecreasing function satisfying $p_{m}(a) \leq g(a)=h(a)$. Therefore applying the case of $m=1$ (which is yet proved) to functions $h(x)$ and $p_{m}(x)$, we obtain

$$
\int_{a}^{b} \frac{1}{\bar{g}^{\prime}} \leq \int_{a}^{b} \frac{1}{h^{\prime}} .
$$

Combining inequalities (3.4) and (3.5) we obtain the desired inequality (3.3). Finally, if equality holds in (3.3), then equalities also hold in (3.4) and (3.5). This gives $g=h=\bar{g}$ and the proof is completed. 


\section{Proof of Proposition 3.1.}

We first prove (3.1) in the case $g(x)<\phi(x)$ for every $x \in(a, b)$. To do this, for every positive integer $n$, consider a partition of the interval $(a, b]$ in $2^{n}$ subintervals of the same length. That is

$$
(a, b]=\bigcup_{k=1}^{2^{n}}\left(x_{k-1, n}, x_{k, n}\right] ; \text { where } x_{k, n}=a+k \frac{b-a}{2^{n}} ; \quad 0 \leq k \leq 2^{n} .
$$

Consider now the $2^{n}$ lines which are tangent to the graphic of the function $y=\phi(x)$ at $x_{k, n}, 1 \leq k \leq 2^{n}$. That is

$$
p_{k, n}(x):=\phi^{\prime}\left(x_{k, n}\right)\left(x-x_{k, n}\right)+\phi\left(x_{k, n}\right), a \leq x \leq b, \quad 1 \leq k \leq 2^{n} .
$$

Define

$$
g_{n}(x):=\max \left\{g(x), p_{1, n}(x), p_{2, n}(x), \ldots, p_{2^{n}, n}(x)\right\}, a \leq x \leq b .
$$

Note that the convexity of $\phi$ gives $g_{n}(x) \leq \phi(x)$ for every $a \leq x \leq b$, $n \geq 1$.

We claim that $g_{n} \rightarrow \phi$ in $L^{\infty}(a, b)$ as $n \rightarrow \infty$. To do this, take an arbitrary $x \in(a, b)$. Then, for fixed $n \geq 1$, there exists $1 \leq k \leq 2^{n}$ such that $x_{k-1, n}<x \leq x_{k, n}$. Using the convexity and monotonicty of $\phi$ we deduce

$$
\begin{gathered}
\phi(x) \geq g_{n}(x) \geq p_{k, n}(x) \geq p_{k, n}\left(x_{k-1, n}\right)=\phi^{\prime}\left(x_{k, n}\right)\left(x_{k-1, n}-x_{k, n}\right)+\phi\left(x_{k, n}\right) \\
\geq \phi^{\prime}(b)\left(x_{k-1, n}-x_{k, n}\right)+\phi(x)=-\phi^{\prime}(b) \frac{b-a}{2^{n}}+\phi(x) .
\end{gathered}
$$

This gives $\left\|\phi-g_{n}\right\|_{L^{\infty}(a, b)} \leq \phi^{\prime}(b) \frac{b-a}{2^{n}}$ and the claim is proved.

Now fix $n_{0}>1$ and consider $a_{0}=a+(b-a) / 2^{n_{0}}$ and $b_{0}=b-(b-a) / 2^{n_{0}}$. Note that $a_{0}=x_{2^{n-n_{0}, n}}$ and $b_{0}=x_{2^{n}-2^{n-n_{0}, n}}$ for every $n \geq n_{0}$. Since $\left[a_{0}, b_{0}\right] \subset(a, b)$ and $g<\phi$ in $(a, b)$, we deduce that there exists $\varepsilon_{0}>0$ (depending on $n_{0}$ ) such that $g(x)<\phi(x)-\varepsilon_{0}$ for every $x \in\left[a_{0}, b_{0}\right]$. Using $g_{n} \rightarrow \phi$ in $L^{\infty}\left(a_{0}, b_{0}\right)$ we can assert that there exists $n_{1} \geq n_{0}$ (depending on $\left.\varepsilon_{0}\right)$ such that $g(x)<g_{n}(x)$ for every $x \in\left[a_{0}, b_{0}\right]$ and $n_{1} \geq n_{0}$. Then

$$
g_{n}(x)=\max \left\{p_{1, n}(x), p_{2, n}(x), \ldots, p_{2^{n}, n}(x)\right\}, a_{0} \leq x \leq b_{0}, n \geq n_{1} .
$$

Consider $n \geq n_{1}$ and $2^{n-n_{0}}<k \leq 2^{n}-2^{n-n_{0}}$. Take $x \in\left[x_{k-1, n}, x_{k, n}\right]$. The convexity of $\phi$ yields $g_{n}(x)=\max \left\{p_{k-1, n}(x), p_{k, n}(x)\right\}$ and consequently $g_{n}^{\prime}(x) \leq \phi^{\prime}\left(x_{k, n}\right)$. This gives

$$
\int_{x_{k-1, n}}^{x_{k, n}} \frac{1}{g_{n}^{\prime}} \geq \frac{x_{k, n}-x_{k-1, n}}{\phi^{\prime}\left(x_{k, n}\right)} .
$$

Therefore, applying Lemma 3.3 in the interval $[a, b]$ it follows that 
$\int_{a}^{b} \frac{1}{g^{\prime}} \geq \int_{a}^{b} \frac{1}{g_{n}^{\prime}} \geq \int_{a_{0}}^{b_{0}} \frac{1}{g_{n}^{\prime}}=\sum_{k=2^{n-n_{0}+1}}^{2^{n}-2^{n-n_{0}}} \int_{x_{k-1, n}}^{x_{k, n}} \frac{1}{g_{n}^{\prime}} \geq \sum_{k=2^{n-n_{0}+1}}^{2^{n}-2^{n-n_{0}}} \frac{x_{k, n}-x_{k-1, n}}{\phi^{\prime}\left(x_{k, n}\right)}$, for every $n \geq n_{1}$. Since $1 / \phi^{\prime}$ is continuous in $\left[a_{0}, b_{0}\right]$ and $x_{k, n}-x_{k-1, n}=$ $(b-a) / 2^{n}$ we deduce that the right term of the last inequality tends to $\int_{a_{0}}^{b_{0}} 1 / \phi^{\prime}$ as $n$ tends to $\infty$. Thus,

$$
\int_{a}^{b} \frac{1}{g^{\prime}} \geq \int_{a_{0}}^{b_{0}} \frac{1}{\phi^{\prime}}
$$

Finally, since $n_{0}>1$ is arbitrary we conclude (3.1) for the case $g<\phi$ in $(a, b)$.

We now turn out to the general case $g \leq \phi$ in $(a, b)$ and we proceed to show (3.1). For this purpose, consider the open set $G=\{x \in(a, b): \phi(x)>g(x)\}$. If $G=\emptyset$, then (3.1) is trivial. Otherwise, $G$ is the countable (possible finite) disjoint union of open intervals. That is, $G=\cup_{n \in X}\left(a_{n}, b_{n}\right)$, where $X \subset \mathbb{N}$, $\phi\left(a_{n}\right)=g\left(a_{n}\right), \phi\left(b_{n}\right) \geq g\left(b_{n}\right)$ and $\phi>g$ in $\left(a_{n}, b_{n}\right)$ for every $n \in X$. Applying the previous case in each interval $\left(a_{n}, b_{n}\right)$ we conclude

$$
\int_{a}^{b} \frac{1}{g^{\prime}}-\int_{a}^{b} \frac{1}{\phi^{\prime}}=\int_{G}\left(\frac{1}{g^{\prime}}-\frac{1}{\phi^{\prime}}\right)=\sum_{n \in X} \int_{a_{n}}^{b_{n}}\left(\frac{1}{g^{\prime}}-\frac{1}{\phi^{\prime}}\right) \geq 0 .
$$

It remains to prove that equality holds in (3.1) if and only if $g=\phi$. To this end suppose that we have equality in (3.1) for some $g$. Take an arbitrary $x_{0} \in[a, b]$ and consider the function

$$
g_{x_{0}}:=\max \left\{g(x), \phi^{\prime}\left(x_{0}\right)\left(x-x_{0}\right)+\phi\left(x_{0}\right)\right\}, a \leq x \leq b .
$$

Clearly $g_{x_{0}}$ is nondecreasing and satisfies $g \leq g_{x_{0}} \leq \phi$ in $[a, b]$ and $g_{x_{0}}(a)=$ $g(a)=\phi(a)$. Hence

$$
\int_{a}^{b} \frac{1}{g_{x_{0}}^{\prime}} \geq \int_{a}^{b} \frac{1}{\phi^{\prime}}=\int_{a}^{b} \frac{1}{g^{\prime}}
$$

Applying Lemma 3.3 yields $g=g_{x_{0}}$ in $[a, b]$. In particular $g\left(x_{0}\right)=$ $g_{x_{0}}\left(x_{0}\right)=\max \left\{g\left(x_{0}\right), \phi\left(x_{0}\right)\right\}=\phi\left(x_{0}\right)$. Since $x_{0} \in[a, b]$ is arbitrary we conclude that $g=\phi$ in $[a, b]$ and the proposition follows.

\section{Proof of Theorem 1.3.}

Obviously, taking $h=\Psi$ in Theorem 1.2 it follows immediately i) $\Rightarrow$ ii) $\Rightarrow$ iii').

It remains to prove iii') $\Rightarrow$ i). Suppose $\int_{1}^{\infty} \frac{1}{\Psi^{\prime}}=\infty$. Using again Theorem 1.2 what is left is to show that $\int_{1}^{\infty} \frac{1}{h^{\prime}}=\infty$, for every nondecreasing function $0 \leq h \in C([1, \infty))$ satisfying $h \leq \Psi$ in $[1, \infty)$. 
To obtain a contradiction suppose that there exists a nondecreasing function $0 \leq h \in C([1, \infty))$ satisfying $h \leq \Psi$ in $[1, \infty)$ and $\int_{1}^{\infty} \frac{1}{h^{\prime}}<\infty$. We first claim that $\lim _{x \rightarrow \infty} h(x) / x=+\infty$. Conversely, suppose that there exist $M>0$ and a divergent sequence $\left\{R_{n}\right\}$ such that $h\left(R_{n}\right) \leq M R_{n}$ for every positive integer $n$. Applying Cauchy-Shwartz inequality we obtain

$$
\begin{gathered}
R_{n}-1=\int_{1}^{R_{n}} \sqrt{h^{\prime}} \frac{1}{\sqrt{h^{\prime}}} \leq\left(\int_{1}^{R_{n}} h^{\prime}\right)^{1 / 2}\left(\int_{1}^{R_{n}} \frac{1}{h}\right)^{1 / 2} \\
=\left(h\left(R_{n}\right)-h(1)\right)^{1 / 2}\left(\int_{1}^{R_{n}} \frac{1}{h^{\prime}}\right)^{1 / 2} \leq\left(M R_{n}\right)^{1 / 2}\left(\int_{1}^{\infty} \frac{1}{h^{\prime}}\right)^{1 / 2},
\end{gathered}
$$

which contradicts that $\left\{R_{n}\right\}$ diverges.

Consequently there exists $R_{1}:=\min \left\{R \geq R_{0}: h(R)=\Psi^{\prime}\left(R_{0}\right)\left(R-R_{0}\right)+\Psi\left(R_{0}\right)\right\}$.

For every $R>R_{1}$ define $g_{R}:\left[R_{0}, R\right] \rightarrow \mathbb{R}$ by

$$
g_{R}(x):= \begin{cases}\Psi^{\prime}\left(R_{0}\right)\left(x-R_{0}\right)+\Psi\left(R_{0}\right) & \text { if } R_{0} \leq x \leq R_{1} \\ h(x) & \text { if } R_{1}<x \leq R\end{cases}
$$

It is easily seen that $g_{R} \in C\left(\left[R_{0}, R\right]\right)$ is a nondecreasing function satisfying $g_{R}\left(R_{0}\right)=\Psi\left(R_{0}\right)$ and $g_{R} \leq \Psi$ in $\left[R_{0}, R\right]$. Then we can apply Proposition 3.1 in the interval $\left[R_{0}, R\right]$ and obtain

$$
\int_{R_{0}}^{R} \frac{1}{\Psi^{\prime}} \leq \int_{R_{0}}^{R} \frac{1}{g_{R}^{\prime}}
$$

Hence, for arbitrary $R>R_{1}$, we have

$$
\int_{R_{1}}^{R} \frac{1}{h^{\prime}}=\int_{R_{0}}^{R} \frac{1}{g_{R}^{\prime}}-\int_{R_{0}}^{R_{1}} \frac{1}{g_{R}^{\prime}} \geq\left(\int_{R_{0}}^{R} \frac{1}{\Psi^{\prime}}\right)-\frac{\left(R_{1}-R_{0}\right)}{\Psi^{\prime}\left(R_{0}\right)} .
$$

Since $\int_{R_{0}}^{\infty} \frac{1}{\Psi^{\prime}}=\infty$, we can take limit as $R$ tends to infty, obtaining $\int_{R_{1}}^{\infty} \frac{1}{h^{\prime}} \geq+\infty$. This contradicts our assumption $\int_{1}^{\infty} \frac{1}{h^{\prime}}<\infty$.

\section{REFERENCES}

[1] G. Alberti, L. Ambrosio, X. Cabré, On a long-standing conjecture of E. De Giorgi: symmetry in $3 D$ for general nonlinearities and a local minimality property, Acta Appl. Math. 65 (2001), 9-33.

[2] L. Ambrosio, X. Cabré, Entire solutions of semilinear elliptic equations in $\mathbb{R}^{3}$ and a conjecture of De Giorgi, J. Amer. Math. Soc. 13 (2000), 725-739.

[3] M. T. Barlow, On the Liouville property for divergence form operators, Canad. J. Math. 50 (1998), 487-496.

[4] H. Berestycki, L. Caffarelli, L. Nirenberg, Further qualitative properties for elliptic equations in unbounded domains, Ann. Scuola Norm. Sup. Pisa Cl. Sci. 25 (1997), 69-94. 
[5] E. De Giorgi, Convergence problems for functionals and operators, Proc. Int. Meeting on Recent Methods in Nonlinear Analysis (Rome, 1978), Pitagora, Bologna, 1979, 131-188.

[6] M. del Pino, M. Kowalczyk, J. Wei, On De Giorgi's conjecture in dimension $N \geq 9$, Ann. of Math. 174 (2011), 1485-1569.

[7] F. Gazzola, The sharp exponent for a Liouville-type theorem for an elliptic inequality, Rend. Istit. Mat. Univ. Trieste 34 (2002), 99-102.

[8] N. Ghoussoub, C. Gui, On a conjecture of De Giorgi and some related problems, Math. Ann. 311 (1998), 481-491.

[9] A. Moradifam, Sharp counterexamples related to the De Giorgi conjecture in dimensions $4 \leq n \leq 8$, Proc. Amer. Math. Soc. 142 (2014), 199-203.

[10] L. Moschini, New Liouville theorems for linear second order degenerate elliptic equations in divergence form, Ann. Inst. H. Poincar Anal. Non Linaire 22 (2005), 11-23.

[11] M. H. Protter, H. F. Weinberger, Maximum principles in differential equations, Prentice-Hall, Englewood Cliffs, N.J. (1967).

[12] S. Villegas, Optimal power in Liouville theorems, preprint, arXiv: 2003.04400 (2020).

Departamento de Análisis Matemático, Universidad de Granada, 18071 Granada, SPAIN.

E-mail address: svillega@ugr.es 